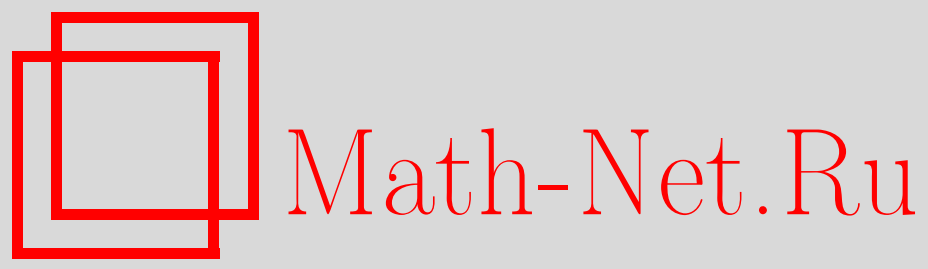

В. В. Жиков, K технике усреднения вариационных задач, Функи. анализ и его прил., 1999, том 33, выпуск $1,14-29$

DOI: https://doi.org/10.4213/faa335

Использование Общероссийского математического портала MathNet.Ru подразумевает, что вы прочитали и согласны с пользовательским соглашением

http://www.mathnet.ru/rus/agreement

Параметры загрузки:

IP : 3.93.64.190

26 апреля 2023 г., $17: 34: 27$

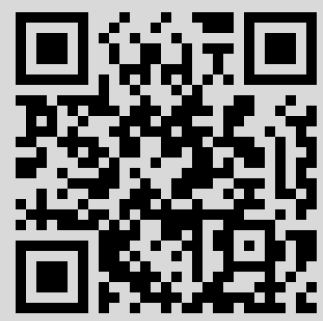




\title{
К технике усреднения вариационных задач
}

\author{
(c) 1999. В. В. Жиков
}

\section{§1. Введение}

1. Пусть $\mu$ - неотрицательная мера на $\mathbb{R}^{N}$, периодическая по каждому переменному $x_{1}, \ldots, x_{N}$ и нормированная условием

$$
\int_{\square} d \mu=1, \quad \text { где } \square=[0,1)^{N}=T^{N} \text { — тор или ячейка периодичности. }
$$

Определим меру $\mu_{\varepsilon}$ равенством

$$
\mu_{\varepsilon}(A)=\varepsilon^{N} \mu\left(\varepsilon^{-1} A\right),
$$

где $A$ - борелевское множество из $\mathbb{R}^{N}$ и $\varepsilon^{-1} A=\left\{\varepsilon^{-1} x, x \in A\right\}$. Мера $\mu_{\varepsilon}$ имеет период $\varepsilon$ и

$$
\mu_{\varepsilon}(\varepsilon \square)=\varepsilon^{N} \int_{\square} d \mu=\varepsilon^{N} ;
$$

поэтому при $\varepsilon \rightarrow 0$ она слабо сходится к мере Лебега: $\mu_{\varepsilon} \rightarrow d x$, т.е.

$$
\lim _{\varepsilon \rightarrow 0} \int_{\mathbb{R}^{N}} \varphi d \mu_{\varepsilon}=\int_{\mathbb{R}^{N}} \varphi d x \quad \forall \varphi \in C_{0}^{\infty}\left(\mathbb{R}^{N}\right) .
$$

Пусть также имеется лагранжиан $f(x, \xi)$, 1-периодический и $\mu$-измеримый по $x \in \mathbb{R}^{N}$, выпуклый по $\xi \in \mathbb{R}^{N}$ и подчиненный степенной оценке

$$
|\xi|^{p} \leqslant f(x, \xi) \leqslant c|\xi|^{p}+1 \text { с некоторым показателем } p>1
$$

и условию $f(x, 0) \equiv 0$. В частности, можно взять не зависящий от $x$ лагранжиан $|\xi|^{p}$.

Рассмотрим задачу Дирихле

$$
m_{\varepsilon}=\inf _{u \in C_{0}^{\infty}(\Omega)} \int_{\Omega}\left(f\left(\varepsilon^{-1} x, \nabla u\right)+|u|^{p}-g u\right) d \mu_{\varepsilon}, \quad g \in C^{\infty}(\bar{\Omega}),
$$

где $\Omega$ - ограниченная липшицева область в $\mathbb{R}^{N}$, а $\bar{\Omega}$ - ее замыкание.

После «естественного замыкания» множества $C_{0}^{\infty}(\Omega)$, позволяющего инфимум заменить на минимум, решение $u^{\varepsilon}$ этой задачи существует и единственно; см. $\S 2$. Ожидаемое свойство усреднения состоит в том, что при $\varepsilon \rightarrow 0$ энергии $m_{\varepsilon}$ и решения $u^{\varepsilon}$ сходятся к энергии $m_{0}$ и решению $u^{0}$ задачи Дирихле, связанной уже с мерой Лебега,

$$
m_{0}=\inf _{u \in C_{0}^{\infty}(\Omega)} \int_{\Omega}\left(f_{0}(\nabla u)+|u|^{p}-g u\right) d x .
$$

\footnotetext{
* Работа выполнена при финансовой поддержке РФФИ, проект № 96-01-00503.
} 
Здесь $f_{0}(\xi)$ - усредненный лагранжиан, определяемый с помощью вариационной задачи на ячейке периодичности,

$$
f_{0}(\xi)=\inf _{u \in C_{\text {per }}^{\infty}(\square)} \int_{\square} f(x, \xi+\nabla u) d \mu,
$$

где $C_{\text {per }}^{\infty}(\square)$ - множество всех бесконечно дифференцируемых периодических функций.

Лагранжиан $f_{0}$ всегда выпуклый и подчинен очевидной оценке $0 \leqslant f_{0}(\xi) \leqslant$ $c|\xi|^{p}+1$. Во многих случаях (но не всегда) он также и коэриитивен: $f_{0}(\xi) \geqslant$ $c_{0}|\xi|^{p}, c_{0}>0$.

Классическая теория усреднения вариационных задач имеет дело с простейшим случаем $d \mu=d x$. Подходящим выбором меры $\mu$ получаются и другие хорошо известные постановки: об усреднении в перфорированных областях, об усреднении вырождающихся эллиптических уравнений, об эффективной проводимости электрических цепей и др.

В настоящей работе изложена техника, позволяющая свести все условия усреднения к одному очень прозрачному свойству меры $\mu$, которое к тому же и необходимо для усреднения. В конкретных постановках это дает возможность обосновать усреднение проше и при более широких условиях, чем это было сделано ранее другими методами.

ОПРЕДЕЛЕНИЕ 1.1. Периодическая мера $\mu$ называется $p$-связной на торе nериодичности, если $u \equiv$ const $\mu$-п.в., как только найдутся $u_{n} \in C_{\mathrm{per}}^{\infty}(\square)$, такие, что

$$
\lim _{n \rightarrow \infty} \int_{\square}\left|u_{n}-u\right|^{p} d \mu=0, \quad \lim _{n \rightarrow \infty} \int_{\square}\left|\nabla u_{n}\right|^{p} d \mu=0 .
$$

Очевидно, что мера Лебега $d x$-связна: функция $u$ из (1.6) принадлежит соболевскому пространству $W^{1, p}$ на торе, имеет нулевой градиент и поэтому есть константа п.в. В общем случае $p$-связность меры $\mu$ означает, что функция $u$ есть константа $\mu$-п.в., если она принадлежит соболевскому пространству $W^{1, p}\left(T^{N}, d \mu\right)$ и обладает нулевым «градиентом» (см. $\left.\S 2\right)$.

Теперь можно сформулировать и теорему усреднения.

Ниже мы используем следующие сокращения:

$$
\langle\varphi\rangle=\int_{\square} \varphi d \mu, \quad C_{\mathrm{per}}^{\infty}=C_{\mathrm{per}}^{\infty}(\square) .
$$

ТЕОРемА 1.2 (теорема усреднения). Пусть мера $\mu$ является $p$-связной, $u^{\varepsilon}$ - решение задачи (1.3) и $u^{0}$ - решение усредненной задачи (1.4). Тогда при $\varepsilon \rightarrow 0$ имеют место

(i) сходимость решений:

$$
\int_{\Omega} \varphi u^{\varepsilon} d \mu_{\varepsilon} \rightarrow \int_{\Omega} \varphi u^{0} d x \quad \forall \varphi \in C^{\infty}(\bar{\Omega}) ;
$$

(ii) сходимость энергий: $m_{\varepsilon} \rightarrow m_{0}$, а такюее

$$
\int_{\Omega} f\left(\varepsilon^{-1} x, \nabla u^{\varepsilon}\right) d \mu_{\varepsilon} \rightarrow \int_{\Omega} f_{0}\left(\nabla u^{0}\right) d x, \quad \int_{\Omega}\left|u^{\varepsilon}\right|^{p} d \mu_{\varepsilon} \rightarrow \int_{\Omega}\left|u^{0}\right|^{p} d x .
$$


При этом усредненньй лагранжиан $f_{0}$, вообще говоря, не коэриитивен.

Квадратичный вариант теоремы $1.2(p=2)$ доказан в [1]; там же замечено, что 2-связность необходима для усреднения.

2. Рассмотрим кратко некоторые варианты задачи усреднения, интересуясь, кроме p-связности соответствующей меры, еще и коэрцитивностью усредненного лагранжиана. В связи с последним вопросом сделаем одно замечание. Для частного случая $f(x, \xi)=|\xi|^{p}$ усредненный лагранжиан обозначим через $\Phi$,

$$
\Phi(\xi)=\inf _{u \in C_{\mathrm{per}}^{\infty}(\square)}\left\langle|\xi+\nabla u|^{p}\right\rangle .
$$

Так как из условия (1.2) следует оценка

$$
\Phi \leqslant f_{0} \leqslant c \Phi+1
$$

то вопросы коэрцитивности достаточно рассмотреть только для $\Phi$.

(1) Вырождающиеся эллиптические уравнения. Пусть мера $\mu$ абсолютно непрерывна относительно меры Лебега, $d \mu=\rho(x) d x$. Тогда она будет $p$-связной, а лагранжиан $\Phi$ - коэрцитивным, если $\int_{\square} \rho^{-1 /(p-1)} d x=\alpha<\infty$.

ДокАЗАТЕльство. Из (1.6) получим по неравенству Гёльдера, что

$$
\int_{\square}\left|\nabla u_{n}\right| d x \leqslant\left(\int_{\square} \rho^{-1 /(p-1)} d x\right)^{(p-1) / p}\left(\int_{\square}\left|\nabla u_{n}\right|^{p} \rho d x\right)^{1 / p} \rightarrow 0
$$

и аналогично $\int_{\square}\left|u-u_{n}\right| d x \rightarrow 0$. Из свойств обычного соболевского пространства $W^{1,1}$ на торе следует, что $u \equiv$ const, т. е. доказана $p$-связность. Далее, две функции $\left(|\xi|^{p} / p\right) \rho,\left(|\eta|^{p^{\prime}} / p^{\prime}\right) \rho^{-1 /(p-1)}$ взаимно сопряжены (здесь и далее $\left.p^{\prime}=p /(p-1)\right)$ и по неравенству Юнга

$$
\begin{gathered}
|\xi+\nabla u|^{p} \rho \geqslant p \eta \cdot(\xi+\nabla u)-(p-1)|\eta|^{p^{\prime}} \rho^{-1 /(p-1)} \quad \forall \eta \in \mathbb{R}^{N}, \quad u \in C_{\mathrm{per}}^{\infty}, \\
\int_{\square}|\xi+\nabla u|^{p} \rho d x \geqslant p \eta \cdot \xi-\alpha(p-1)|\eta|^{p^{\prime}}
\end{gathered}
$$

Беря справа в (1.9) супремум по $\eta \in \mathbb{R}^{N}$, а слева инфимум по $u \in C_{\text {per }}^{\infty}$, получим оценку $\Phi(\xi) \leqslant p \alpha^{1-p}|\xi|^{p}$. Для сравнения отметим, что ранее [2] для доказательства теоремы усреднения на вес $\rho$ налагалось весьма сложное условие принадлежности так называемому $A_{p}$-классу Макенхаупта.

(2) Усреднение в перфорированных областях. Пусть $Q$ - открытое периодическое множество в $\mathbb{R}^{N}$ (например, внешность периодической системы шаров) и

$$
d \mu=\rho d x, \quad \rho(x)= \begin{cases}|\square \cap Q|^{-1} & \text { в } Q, \\ 0 & \text { вне } Q .\end{cases}
$$

В данном случае $d \mu_{\varepsilon}=\rho\left(\varepsilon^{-1} x\right) d x$ и интегрирование в (1.3) ведется только по $\varepsilon Q \cap \Omega$ - «перфорированной области».

Усреднению в перфорированных областях посвящена обширная литература, см. [3-7]. Классический метод основан на идее продолжения решения 
с перфорированного пространства на все $\Omega$ с сохранением энергетических оценок, что требовало существенных ограничений на структуру периодического множества $Q$ (условие «сильной связанности» по Хруслову). Так, давний вопрос об обосновании усреднения в случае, когда $Q$ просто связно в $\mathbb{R}^{N}$, не поддавался решению (важный пример: $Q$ есть внешность плотной кубической упаковки шаров в $\mathbb{R}^{3}$ ).

Между тем обычная связность множества $Q$ в $\mathbb{R}^{N}$ (и даже только на торе) влечет за собой $p$-связность меры $\mu$, что непосредственно ясно из определения 1.1. Значит, в этом случае теорема усреднения действует. Доказано также [7], что связность в $\mathbb{R}^{N}$ обеспечивает коэрцитивность $\Phi$. Отметим, что для $p$-связности меры (1.10) вовсе не обязательно, чтобы множество $Q$ было связным в $\mathbb{R}^{N}$ или хотя бы на торе. Эти вопросы обсуждаются в [1], в частности, рассмотрен пример, когда множество $Q$ связно на торе (значит, соответствуюшая мера $p$-связна), несвязно в $\mathbb{R}^{N}$, а усредненный лагранжиан $\Phi$ коэрцитивен.

(3) Фрактальные меры. Через $C$ обозначим классическое канторово множество на $[0,1]$ (делим отрезок на три равных, средний открытый промежуток выбрасываем и т.д.), и пусть $\mu_{0}$ - естественная нормированная мера, сосредоточенная на $C$. На квадрате $\square=[0,1)^{2}$ определим меру $\mu$ равенством

$$
d \mu=\frac{d \mu_{0}\left(x_{1}\right) \times d x_{2}+d x_{1} \times d \mu_{0}\left(x_{2}\right)}{2}
$$

и продолжим ее периодически на $\mathbb{R}^{2}$. По определению мера $\mu$ сосредоточена на каркасе $C \times[0,1) \cup[0,1) \times C$. Доказано [1], что эта мера $p$-связна для любого $p \geqslant 1$.

(4) Периодические графы. Рассмотрим на плоскости периодический связный граф, например, обычную квадратную сетку. Меру $\mu$ определим как сумму линейных мер звеньев, взятых с некоторыми положительными весами. Тогда свойство p-связности будет выполнено. В приложениях часто указанные веса случайны, но усреднение случайных мер - особая тема, которую мы здесь не затрагиваем.

Для чтения настоящей работы не требуется никаких сведений из теории усреднения. В целом изложение вполне элементарно, за исключением, может быть, одной ссылки на теорию двойственности. Впрочем, в важном случае, когда лагранжиан $f$ является $p$-однородным по $\xi$, например, когда $f(x, \xi)=|\xi|^{p}$, мы указываем (см. 4$)$ способ изложения, вообще не использующий двойственности.

Ниже часто используется следующее свойство среднего значения.

Пусть $b(x)$ - заданная на $\mathbb{R}^{N}$ периодическая $\mu$-суммируемая функция. Из (1.1) следует слабая сходимость мер в $\mathbb{R}^{N}: b\left(\varepsilon^{-1} x\right) d \mu_{\varepsilon} \rightarrow\langle b\rangle d x$. Отсюда

$$
\lim _{\varepsilon \rightarrow 0} \int_{\bar{A}} \varphi(x) b\left(\varepsilon^{-1} x\right) d \mu_{\varepsilon}=\lim _{\varepsilon \rightarrow 0} \int_{A} \varphi(x) b\left(\varepsilon^{-1} x\right) d \mu_{\varepsilon}=\langle b\rangle \int_{A} \varphi d x,
$$

где $A$ - ограниченная область, граница которой имеет нулевую лебегову меру и $\varphi$ непрерывна в $\bar{A}$. В частности, $\lim _{\varepsilon \rightarrow 0} \mu_{\varepsilon}(A)$ равен $|A|$, мере Лебега. 


\section{§2. Вспомогательные вопросы. Лемма об аппроксимации}

1. Определим соболевское пространство $W_{0}^{1, p}\left(\Omega, d \mu_{\varepsilon}\right)$ как замыкание множества $\left\{(u, \nabla u)\right.$, где $\left.u \in C_{0}^{\infty}(\Omega)\right\}$ в произведении $L^{p}\left(\Omega, d \mu_{\varepsilon}\right) \times L^{p}\left(\Omega, d \mu_{\varepsilon}\right)^{N}$. Элементами указанного замыкания служат пары $(u, v)$, причем $v$ называется градиентом $u$ и обозначается $\nabla u$. Конечно, привычней первую компоненту $u$ называть функцией из соболевского пространства; тогда эта функция может иметь много градиентов; см. [1]. Впрочем, неединственность градиента особых хлопот не вызывает.

Рассмотрим исходную вариационную задачу (1.3). Очевидно, что

$$
m_{\varepsilon}=\min _{(u, v) \in W^{1, p}\left(\Omega, d \mu_{\varepsilon}\right)} \int_{\Omega}\left(f\left(\varepsilon^{-1} x, v\right)+|u|^{p}-g u\right) d \mu_{\varepsilon},
$$

и если минимум достигается на двух элементах $\left(u_{1}, v_{1}\right)$ и $\left(u_{2}, v_{2}\right)$, то $u_{1}=u_{2}$ в силу строгой выпуклости слагаемого $|u|^{p}$. В теореме усреднения говорится только об энергии $m_{\varepsilon}$ и решении $u^{\varepsilon}$ (а не о его градиенте), но энергия, очевидно, не зависит от выбора градиента решения. Далее, так как $f(x, 0) \equiv 0$, то $m_{\varepsilon} \leqslant 0$ и несложные вычисления дают

$$
\begin{aligned}
\int_{\Omega}\left(f\left(\varepsilon^{-1} x, \nabla u^{\varepsilon}\right)+\left|u^{\varepsilon}\right|^{p}\right) d \mu_{\varepsilon} & \leqslant \int_{\Omega} g u^{\varepsilon} d \mu_{\varepsilon} \leqslant\left(\int_{\Omega}|g|^{p^{\prime}} d \mu_{\varepsilon}\right)^{1 / p^{\prime}}\left(\int_{\Omega}\left|u^{\varepsilon}\right|^{p} d \mu_{\varepsilon}\right)^{1 / p} \\
& \leqslant \int_{\Omega}|g|^{p^{\prime}} d \mu_{\varepsilon} \leqslant C<\infty
\end{aligned}
$$

Заметим, что левая часть формулы (2.1) есть непрерывный функционал на $W_{0}^{1, p}\left(\Omega, d \mu_{\varepsilon}\right)$. Поэтому найдутся

$$
\tilde{u}^{\varepsilon} \in C_{0}^{\infty}(\Omega) \text {, такие, что }\left\|\tilde{u}^{\varepsilon}-u^{\varepsilon}\right\|_{W_{0}^{1, p}\left(\Omega, d \mu_{\varepsilon}\right)} \leqslant \varepsilon, 0 \leqslant \widetilde{m}_{\varepsilon}-m_{\varepsilon} \leqslant \varepsilon,
$$

где

$$
\widetilde{m}_{\varepsilon}=\int_{\Omega}\left(f\left(\varepsilon^{-1} x, \nabla \tilde{u}^{\varepsilon}\right)+\left|\tilde{u}^{\varepsilon}\right|^{p}-g \tilde{u}^{\varepsilon}\right) d \mu_{\varepsilon} .
$$

2. Для строгого определения решения усредненной задачи нам понадобятся простые свойства усредненного лагранжиана $f_{0}$ (см. (1.5)).

ПрЕДЛОЖЕНИЕ 2.1. Существует такое подпространство $E \subset \mathbb{R}^{N}$, ито

(i) $f_{0}(\xi+q)=f_{0}(\xi)$ для любих $\xi \in \mathbb{R}^{N}, q \in E^{\perp}$;

(ii) $c_{0}|\xi|^{p} \leqslant f_{0}(\xi) \leqslant c|\xi|^{p}+1 \quad \partial \Omega \Omega \quad \xi \in E \quad u c_{0}>0$.

Кроме того, $f_{0}(0)=0$. Другими словами, функция $f_{0}$ есть прямал сумма коэрцитивной функции на $E$ и нулевой функции на ортогональном дополнении.

ДокаЗАТЕЛЬСТво. Очевидно, что $Q=\left\{q \in \mathbb{R}^{N}, \Phi(q)=0\right\}$ есть подпространство в $\mathbb{R}^{N}$. Для $\xi \in \mathbb{R}^{N}, q \in Q$ по неравенству (1.8)

$0 \leqslant f_{0}(\xi+t q) \leqslant c \Phi(\xi+t q)+1 \leqslant 2^{p} c[\Phi(\xi)+\Phi(t q)]+1=2^{p} c \Phi(\xi)+1 \quad \forall t \in \mathbb{R}^{1}$.

Отсюда следует, что выпуклая функция $\varphi(t)=f_{0}(\xi+t q)$ является константой, равной $f_{0}(\xi)$, что доказывает свойство (i). В частности, $f_{0}(q)=f_{0}(0)=0$ 
(последнее равенство есть следствие условия $f(x, 0)=0$ ). Кроме того, функция $\Phi$ коэрцитивна на $E=Q^{\perp}: c_{0}|\xi|^{p} \leqslant \Phi(\xi) \leqslant|\xi|^{p}, c_{0}>0$. Отсюда и из соотношений (1.8) получаем утверждение (ii). Предложение доказано.

Для простоты будем представлять себе, что $E$ - координатное подпространство,

$$
E=\mathbb{R}^{k}=\left\{\xi \in \mathbb{R}^{N}, \xi=\left(\xi_{1}, \ldots, \xi_{k}, 0, \ldots, 0\right)\right\} .
$$

Примером некоэрцитивной функции служит $\left(\xi_{1}^{2}+\cdots+\xi_{k}^{2}\right)^{p / 2}$. Коэрцитивному лагранжиану $f_{0}$ соответствуют классические соболевские пространства $W^{1, p}(\Omega)$ и $W_{0}^{1, p}(\Omega)$, а некоэрцитивному - пространство

$$
S=\left\{u \in L^{p}(\Omega), \frac{\partial u}{\partial x_{1}}, \ldots, \frac{\partial u}{\partial x_{k}} \in L^{p}(\Omega)\right\} \quad \text { с нормой }\|u\|_{L^{p}}+\sum_{j=1}^{k}\left\|\frac{\partial u}{\partial x_{j}}\right\|_{L^{p}}
$$

и его подпространство $S_{0}=\left\{u \in S,\left.u\right|_{\partial \Omega}=0\right\}$. При этом равенство $\left.u\right|_{\partial \Omega}=0$ означает по определению, что

$$
\int_{\Omega} u \frac{\partial \varphi}{\partial x_{j}} d x=-\int_{\Omega} \frac{\partial u}{\partial x_{j}} \varphi d x \quad \forall \varphi \in C(\bar{\Omega})(j=1, \ldots, k) .
$$

Важно, что множество $C_{0}^{\infty}(\Omega)$ плотно в $S_{0}$. Для звездной области $\Omega$ этот факт доказывается обычным сглаживанием, а для липшицевой требуются более изощренные рассуждения (см. [8, с. 311]). Итак, усредненная задача (1.4) имеет единственное решение в $S_{0}$.

3. Перейдем к обсуждению некоторых свойств периодических задач.

Для $a \in L_{\mathrm{per}}^{1}(\square, d \mu), b \in L_{\mathrm{per}}^{1}(\square, d \mu)^{N}$ запись $a=\operatorname{div} b$ означает по определению

$$
\langle a \varphi\rangle=-\langle b \cdot \nabla \varphi\rangle \quad \forall \varphi \in C_{\text {per }}^{\infty}(\square) .
$$

$\mathrm{B}$ частности, если $\operatorname{div} b=0$, то вектор $b$ мы называем соленоидальныл.

Определим соболевское пространство периодических функций $W_{\mathrm{per}}^{1, p}(\square, d \mu)$ как замыкание множества $\left\{(u, \nabla u)\right.$, где $\left.u \in C_{\text {per }}^{\infty}(\square)\right\}$ в произведении $L^{p}(\square, d \mu) \times L^{p}(\square, d \mu)^{N}$.

Ясно, что задача

$$
\min _{(u, v) \in W^{1, p}(\square, d \mu)}\left\langle|v|^{p}+|u|^{p}-\frac{g u}{p}\right\rangle, \quad g \in L^{p^{\prime}}(\square, d \mu),
$$

имеет единственное решение $(u, \nabla u)$ и для него выполнено уравнение Эйлера

$$
\left\langle|\nabla u|^{p-2} \nabla u \cdot \nabla \varphi+|u|^{p-2} u \varphi\right\rangle=\langle g \varphi\rangle \quad \forall \varphi \in C_{\mathrm{per}}^{\infty}(\square),
$$

записываемое кратко как $-\operatorname{div}\left(|\nabla u|^{p-2} \nabla u\right)+|u|^{p-2} u=g$.

4. Определение $p$-связности можно перефразировать следующим образом: мера $\mu$ р-связна на торе периодичности, если

$$
(u, 0) \in W^{1, p}(\square, d \mu) \Longrightarrow u=\text { const } \mu-n . \text { в. }
$$

Сейчас мы установим одно важное следствие $p$-связности. 
ЛЕмма 2.2 (лемма об аппроксимации). Пусть мера $\mu$ является $p$-связной и $a \in L^{p^{\prime}}(\square, d \mu),\langle a\rangle=0$. Тогда для любого $\delta>0$ найдутся $a_{0}, b_{1}, \ldots, b_{N} \in$ $L^{p^{\prime}}(\square, d \mu)$, maкие, что $\left\|a_{0}\right\|_{L^{p^{\prime}(\square, d \mu)}} \leqslant \delta, a=a_{0}+\operatorname{div} b$.

ДоказАтельство. Рассмотрим множество $A$ всех функций $a \in L^{p^{\prime}}(\square, d \mu)$, допускающих представление $a=\operatorname{div} b$ с $b \in L^{p^{\prime}}(\square, d \mu)^{N}$. Нам требуется проверить, что

\section{$A$ плотно в $L^{p^{\prime}}(\square, d \mu) / \mathbb{R}^{1}$.}

Допустим, что $\theta \in L^{p}(\square, d \mu),\langle\theta\rangle=0,\langle\theta a\rangle=0$ для любого $a \in A$. Рассмотрим эллиптическую периодическую задачу

$$
-\operatorname{div}\left(|\nabla u|^{p-2} \nabla u\right)+|u|^{p-2} u=|\theta|^{p-2} \theta \in L^{p^{\prime}}(\square, d \mu),
$$

которая, как мы знаем, разрешима в $W^{1, p}(\square, d \mu)$. Очевидно, что $|\theta|^{p-2} \theta-$ $|u|^{p-2} u \in A$ и поэтому

$$
\left\langle|\theta|^{p}\right\rangle=\left\langle|u|^{p-2} u \theta\right\rangle \leqslant\left\langle|u|^{p}\right\rangle^{(p-1) / p}\left\langle|\theta|^{p}\right\rangle^{1 / p}, \quad\left\langle|\theta|^{p}\right\rangle \leqslant\left\langle|u|^{p}\right\rangle .
$$

С другой стороны, из уравнения (2.4) имеем

$$
\left\langle|\nabla u|^{p}\right\rangle+\left\langle|u|^{p}\right\rangle=\left\langle|\theta|^{p-1} u\right\rangle \leqslant\left\langle|\theta|^{p}\right\rangle^{(p-1) / p}\left\langle|u|^{p} \mid\right\rangle^{1 / p}, \quad\left\langle|u|^{p}\right\rangle \leqslant\left\langle|\theta|^{p}\right\rangle .
$$

Отсюда и из соотношений (2.5) следует, что

$$
\left\langle|\theta|^{p}\right\rangle=\left\langle|u|^{p}\right\rangle, \quad\left\langle|\nabla u|^{p}\right\rangle=0 .
$$

В силу $p$-связности $u \equiv$ const; следовательно (см. уравнение (2.4)), $\theta \equiv$ const. Так как $\langle\theta\rangle=0$, то $\theta=0$. Лемма доказана.

Прежде чем из леммы об аппроксимации извлечь «рабочую» лемму, сделаем одно замечание. Пусть имеется соотношение $a=\operatorname{div} b$, т. е. тождество (2.3) на периодических функциях. Тогда для $a_{\varepsilon}=a\left(\varepsilon^{-1} x\right), b_{\varepsilon}=b\left(\varepsilon^{-1} x\right)$ имеем тождество на финитных функциях

$$
\int a_{\varepsilon} \psi d \mu_{\varepsilon}=-\varepsilon \int b_{\varepsilon} \cdot \nabla \psi d \mu_{\varepsilon} \quad \forall \psi \in C_{0}^{\infty}\left(\mathbb{R}^{N}\right) .
$$

Лемма 2.3. Пусть $a \in L^{p^{\prime}}(\square, d \mu),\langle a\rangle=0$. Тогда

$$
\begin{aligned}
w^{\varepsilon} \in C_{0}^{\infty}(\Omega), \quad \varlimsup_{\varepsilon \rightarrow 0} \int_{\Omega}\left(\left|w^{\varepsilon}\right|^{p}+\left|\nabla w^{\varepsilon}\right|^{p}\right) d \mu_{\varepsilon}<\infty \\
\Longrightarrow \lim _{\varepsilon \rightarrow 0} \int_{\Omega} a\left(\varepsilon^{-1} x\right) w^{\varepsilon}(x) d \mu_{\varepsilon}=0 .
\end{aligned}
$$

ДокАЗАТЕльство. Так как по лемме 2.2

$$
\int_{\Omega} a\left(\varepsilon^{-1} x\right) w^{\varepsilon}(x) d \mu_{\varepsilon}=\int_{\Omega} a_{0}\left(\varepsilon^{-1} x\right) w^{\varepsilon}(x) d \mu_{\varepsilon}-\varepsilon \int_{\Omega} b_{i}\left(\varepsilon^{-1} x\right) \frac{\partial w^{\varepsilon}}{\partial x_{i}} d \mu_{\varepsilon}
$$

и по свойству среднего значения

$$
\begin{aligned}
& \lim _{\varepsilon \rightarrow 0} \int_{\Omega}\left|b_{i}\left(\varepsilon^{-1} x\right)\right|^{p^{\prime}} d \mu_{\varepsilon}=|\Omega|\left\langle\left|b_{i}\right|^{p^{\prime}}\right\rangle, \\
& \lim _{\varepsilon \rightarrow 0} \int_{\Omega}\left|a_{0}\left(\varepsilon^{-1} x\right)\right|^{p^{\prime}} d \mu_{\varepsilon}=|\Omega|\left\langle\left|a_{0}\right|^{p^{\prime}}\right\rangle \leqslant|\Omega| \delta,
\end{aligned}
$$


то импликация (2.6) очевидна. Лемма доказана.

5. Отметим некоторые свойства сопряженного с $f_{0}$ лагранжиана

$$
f_{0}^{*}(\eta)=\sup _{\xi \in \mathbb{R}^{N}}\left\{\eta \cdot \xi-f_{0}(\xi)\right\} .
$$

Из предложения 2.1 следует, что

$$
\begin{array}{cl}
-1+\hat{c}_{0}|\eta|^{p^{\prime}} \leqslant f_{0}^{*}(\eta) \leqslant \hat{c}|\eta|^{p^{\prime}} \quad\left(\hat{c}_{0}>0\right) & \text { для } \eta \in E, \\
f_{0}^{*}(\eta)=\infty & \text { для } \eta \notin E .
\end{array}
$$

Нам понадобится также вариационное представление для $f_{0}^{*}$, аналогичное исходному представлению (1.5) для $f_{0}$. С этой целью введем сопряженный с $f(x, \xi)$ лагранжиан

$$
f^{*}(x, \eta)=\sup _{\xi \in \mathbb{R}^{N}}\{\eta \cdot \xi-f(x, \xi)\} .
$$

Тогда из (1.5) и общей теоремы двойственности [3, гл. 11; 4, гл. 14] следует, что

$$
\begin{gathered}
f_{0}^{*}(\eta)=\min _{z \in M(\eta)} \int_{\square} f^{*}(x, z(x)) d \mu, \\
M(\eta)=\left\{z \in L_{\mathrm{per}}^{p^{\prime}}(\square, d \mu)^{N}, \operatorname{div} z=0,\langle z\rangle=\eta\right\} .
\end{gathered}
$$

Из (2.7) ясно, что $M(\eta) \neq \varnothing$ только для $\eta \in E$.

\section{§3. Техника усреднения}

1. Изучим произвольную последовательность

$$
u^{\varepsilon} \in C_{0}^{\infty}(\Omega), \quad \int_{\Omega}\left|u^{\varepsilon}\right|^{p} d \mu_{\varepsilon} \leqslant \alpha<\infty, \quad \int_{\Omega}\left|\nabla u^{\varepsilon}\right|^{p} d \mu_{\varepsilon} \leqslant \beta<\infty .
$$

Сначала рассмотрим последовательность $u^{\varepsilon}$ как таковую, используя условие (3.1) только для $u^{\varepsilon}$. Имеет место следующее простое утверждение.

ЛЕмМа 3.1. Найдутся подпоследовательность (обозначаемая ниже тем же символом $\left.u^{\varepsilon}\right)$ и функция $u^{0} \in L^{p}(\Omega)$, такие, что

$$
\begin{aligned}
& \lim _{\varepsilon \rightarrow 0} \int_{\Omega} \varphi u^{\varepsilon} d \mu_{\varepsilon}=\int_{\Omega} \varphi u^{0} d x \quad \forall \varphi \in C^{\infty}(\bar{\Omega}), \\
& \frac{\lim _{\varepsilon \rightarrow 0} \int_{\Omega}\left|u^{\varepsilon}\right|^{p} d \mu_{\varepsilon} \geqslant \int_{\Omega}\left|u^{0}\right|^{p} d x .}{}
\end{aligned}
$$

ДоказАтельство. Так как $\lim _{\varepsilon \rightarrow 0} \mu_{\varepsilon}(\Omega)=|\Omega|($ см. (1.11)) и

$$
\int_{\bar{\Omega}}\left|u^{\varepsilon}\right| d \mu_{\varepsilon} \leqslant\left(\int_{\Omega}\left|u^{\varepsilon}\right|^{p} d \mu_{\varepsilon}\right)^{1 / p} \mu_{\varepsilon}^{1 / p^{\prime}}(\Omega) \leqslant \alpha \mu_{\varepsilon}^{1 / p^{\prime}}(\Omega)
$$

то последовательность мер $u^{\varepsilon} d \mu_{\varepsilon}$ на $\bar{\Omega}$ слабо компактна; без потери общности ее можно считать слабо сходящейся:

$$
\lim _{\varepsilon \rightarrow 0} \int_{\Omega} \varphi u_{\varepsilon} d \mu_{\varepsilon}=\int_{\bar{\Omega}} \varphi d U \quad \forall \varphi \in C^{\infty}(\bar{\Omega}),
$$


где $U-$ некоторая борелевская мера на $\bar{\Omega}$. Кроме того,

$\left|\int_{\Omega} \varphi u^{\varepsilon} d \mu_{\varepsilon}\right| \leqslant\left(\int_{\Omega}\left|u^{\varepsilon}\right|^{p} d \mu_{\varepsilon}\right)^{1 / p}\left(\int_{\Omega}|\varphi|^{p^{\prime}} d \mu_{\varepsilon}\right)^{1 / p^{\prime}} \leqslant \alpha^{1 / p}\left(\int_{\Omega}|\varphi|^{p^{\prime}} d \mu_{\varepsilon}\right)^{1 / p^{\prime}}$.

Переходя здесь к пределу по свойству среднего значения, получим неравенство

$$
\left|\int_{\bar{\Omega}} \varphi d U\right| \leqslant \alpha^{1 / p}\left(\int_{\Omega}|\varphi|^{p^{\prime}} d x\right)^{1 / p^{\prime}}
$$

из которого ясно, что $d U=u^{0} d x, u^{0} \in L^{p}(\Omega, d x)$. Далее имеем

$$
\begin{aligned}
\frac{1}{p} \int_{\Omega}\left|u^{\varepsilon}\right|^{p} d \mu_{\varepsilon} & \geqslant \int_{\Omega} \varphi u^{\varepsilon} d \mu_{\varepsilon}-\frac{1}{p^{\prime}} \int_{\Omega} \varphi^{p^{\prime}} d \mu_{\varepsilon}, \\
\frac{1}{p} \frac{\lim }{\varepsilon \rightarrow 0} \int_{\Omega}\left|u^{\varepsilon}\right|^{p} d \mu_{\varepsilon} & \geqslant \int_{\Omega} \varphi u^{0} d x-\frac{1}{p^{\prime}} \int_{\Omega}|\varphi|^{p^{\prime}} d x, \quad \varphi \in C^{\infty}(\bar{\Omega}) .
\end{aligned}
$$

Поскольку $C^{\infty}(\bar{\Omega})$ плотно в $L^{p^{\prime}}(\Omega)$, последнее неравенство верно для любого $\varphi \in L^{p^{\prime}}(\Omega)$. Взяв $\varphi=u^{0}\left|u^{0}\right|^{p-2}$, получим (3.3). Лемма доказана.

Возвращаясь к ситуации (3.1), отметим, что заранее совсем не ясно, что предельная функция $u^{0}$ имеет градиент. Это обстоятельство зависит от свойств меры $\mu$, в частности, от наличия достаточного запаса соленоидальных периодических векторов. Справедливо следующее ключевое свойство.

Лемма 3.2. Пусть $z \in L^{p^{\prime}}(\square, d \mu), \operatorname{div} z=0,\langle z\rangle=\eta u z_{\varepsilon}(x)=z\left(\varepsilon^{-1} x\right)$. Тогда $\nabla u^{0} \cdot \eta \in L^{p}(\Omega)$ (в смысле распределений),

$$
\begin{aligned}
\int_{\Omega}\left|\nabla u^{0} \cdot \eta\right|^{p} d x & \leqslant \beta\left\langle|z|^{p^{\prime}}\right\rangle^{p-1}, \\
\lim _{\varepsilon \rightarrow 0} \int_{\Omega} \varphi \nabla u^{\varepsilon} \cdot z_{\varepsilon} d \mu_{\varepsilon} & =\int_{\Omega} \varphi \nabla u^{0} \cdot \eta d x, \quad \varphi \in C^{\infty}(\bar{\Omega}) .
\end{aligned}
$$

Кроме того, $\left.u^{0}\right|_{\partial \Omega}=0$ в том смислле, ито

$$
\int_{\Omega} u^{0} \nabla \varphi \cdot \eta d x=-\int_{\Omega} \nabla u^{0} \cdot \eta \varphi d x \quad \forall \varphi \in C^{\infty}(\bar{\Omega}) .
$$

ДокАЗАТЕльСТво. При достаточно малом $\varepsilon$

$$
\int_{\bar{\Omega}}\left|\nabla u^{\varepsilon} \cdot z_{\varepsilon}\right| d \mu_{\varepsilon} \leqslant\left(\int_{\Omega}\left|\nabla u^{\varepsilon}\right|^{p} d \mu_{\varepsilon}\right)^{1 / p}\left(\int_{\Omega}\left|z_{\varepsilon}\right|^{p^{\prime}} d \mu_{\varepsilon}\right)^{1 / p^{\prime}} \leqslant 2 \beta^{1 / p}|\Omega|^{1 / p^{\prime}}\left\langle|z|^{p^{\prime}}\right\rangle^{1 / p^{\prime}} .
$$

Поэтому мы считаем, что последовательность мер $\nabla u^{\varepsilon} \cdot z_{\varepsilon} d \mu_{\varepsilon}$ слабо сходится:

$$
\lim _{\varepsilon \rightarrow 0} \int_{\Omega} \varphi \nabla u^{\varepsilon} \cdot z_{\varepsilon} d \mu_{\varepsilon}=\int_{\bar{\Omega}} \varphi d V, \quad \varphi \in C^{\infty}(\bar{\Omega}),
$$

где $V$ - борелевская мера на $\bar{\Omega}$. Так как

$$
\left|\int_{\Omega} \varphi \nabla u^{\varepsilon} \cdot z_{\varepsilon} d \mu_{\varepsilon}\right| \leqslant \beta^{1 / p}\left(\int_{\Omega}|\varphi|^{p^{\prime}}\left|z_{\varepsilon}\right|^{p^{\prime}} d \mu_{\varepsilon}\right)^{1 / p^{\prime}},
$$


то после перехода к пределу (по свойству среднего значения) получим неравенство

$$
\left|\int_{\bar{\Omega}} \varphi d V\right| \leqslant \beta^{1 / p}\left(\int_{\Omega}|\varphi|^{p^{\prime}} d x\right)^{1 / p^{\prime}}\left\langle|z|^{p^{\prime}}\right\rangle^{1 / p^{\prime}}
$$

из которого ясно, что $d V=v^{0} d x, v^{0} \in L^{p}(\Omega)$. Итак,

$$
v^{0} \in L^{p}(\Omega), \quad \lim _{\varepsilon \rightarrow 0} \int_{\Omega} \varphi \nabla u^{\varepsilon} \cdot z_{\varepsilon} d \mu_{\varepsilon}=\int_{\Omega} \varphi v^{0} d x \quad \forall \varphi \in C^{\infty}(\bar{\Omega}) .
$$

Теперь проверим, что $v^{0}=\nabla u^{0} \cdot \eta$. Для этого надо доказать равенство

$$
\lim _{\varepsilon \rightarrow 0} \int_{\Omega} \varphi \nabla u^{\varepsilon} \cdot z_{\varepsilon} d \mu_{\varepsilon}=-\int_{\Omega} u^{0} \nabla \varphi \cdot \eta d x
$$

для любого $\varphi \in C^{\infty}(\bar{\Omega})$, что даст одновременно и соотношение $(3.5)$. Из соленоидальности вектора $z(x)$ и финитности $u^{\varepsilon}$ имеем

$\int_{\Omega} \varphi \nabla u^{\varepsilon} \cdot z_{\varepsilon} d \mu_{\varepsilon}=-\int_{\Omega} u^{\varepsilon} z_{\varepsilon} \cdot \nabla \varphi d \mu_{\varepsilon}=-\int_{\Omega} u^{\varepsilon} \nabla \varphi \cdot \eta d \mu_{\varepsilon}-\int_{\Omega} u^{\varepsilon}\left(z_{\varepsilon}-\eta\right) \cdot \nabla \varphi d \mu_{\varepsilon}$.

Замечаем, что предел первого слагаемого справа равен как раз правой части формулы (3.6) (согласно (3.2)). Предел же второго равен нулю - это получается из леммы 2.3 , если взять в ней $a=z_{j}-\eta_{j}, w^{\varepsilon}=u^{\varepsilon} \partial \varphi / \partial x_{j}$.

2. Установим теперь следующее свойство.

Лемма 3.3. Пусть $u^{\varepsilon}$ удовлетворяет условиям (3.1), (3.2). Тогда

$$
\lim _{\varepsilon \rightarrow 0} \int_{\Omega} f\left(\varepsilon^{-1} x, \nabla u^{\varepsilon}\right) d \mu_{\varepsilon} \geqslant \int_{\Omega} f_{0}\left(\nabla u^{0}\right) d x .
$$

ДокАЗАтЕЛЬство. Пусть $\eta \in E=\mathbb{R}^{k}$ и $z(x)$ - решение вариационной задачи (2.8), т.е.

$$
f_{0}^{*}(\eta)=\int_{\square} f^{*}(x, z(x)) d \mu, \quad \operatorname{div} z=0, \quad\langle z\rangle=\eta .
$$

Если $A$ - произвольная липшицева подобласть области $\Omega, \varphi \in C_{0}^{\infty}(A)$, $0 \leqslant \varphi \leqslant 1$, то по неравенству Юнга

$\int_{\bar{A}} f\left(\varepsilon^{-1} x, \nabla u^{\varepsilon}(x)\right) d \mu_{\varepsilon} \geqslant \int_{A} \varphi \nabla u^{\varepsilon}(x) \cdot z\left(\varepsilon^{-1} x\right) d \mu_{\varepsilon}-\int_{A} \varphi f^{*}\left(\varepsilon^{-1} x, z\left(\varepsilon^{-1} x\right)\right) d \mu_{\varepsilon}$.

Отсюда по лемме 3.2 (см. (3.4)) и свойству среднего значения

$$
\varliminf_{\varepsilon \rightarrow 0} \int_{\bar{A}} f\left(\varepsilon^{-1} x, \nabla u^{\varepsilon}(x)\right) d \mu_{\varepsilon} \geqslant \int_{A} \varphi \nabla u^{0} \cdot \eta d x-\int_{A} \varphi f_{0}^{*}(\eta) d x .
$$

Поскольку $\nabla u^{0} \cdot \eta \in L^{p}(\Omega)$,

$$
\frac{\lim _{\varepsilon \rightarrow 0}}{\int_{\bar{A}}} f\left(\varepsilon^{-1} x, \nabla u^{\varepsilon}(x)\right) d \mu_{\varepsilon} \geqslant \int_{A} \nabla u^{0} \cdot \eta d x-\int_{A} f_{0}^{*}(\eta) d x .
$$


Разбивая область $\Omega$ на подобласти и беря различные $\xi \in \mathbb{R}^{k}$, заключаем, что

$$
\varliminf_{\varepsilon \rightarrow 0} J_{\varepsilon} \geqslant \int_{\Omega} \nabla u^{0} \cdot v(x) d x-\int_{\Omega} f_{0}^{*}(v(x)) d x
$$

для любого кусочно постоянного вектора $v(x)$ со значениями в $\mathbb{R}^{k}$. Множество таких векторов $v(x)$ плотно в $\left(L^{p^{\prime}}(\Omega)\right)^{k}$.

Мы заранее знаем, что $\partial u^{0} / \partial x_{1}, \ldots, \partial u^{0} / \partial x_{k} \in L^{p}(\Omega)$; отсюда и из $(2.7)$ заключаем, что правая часть неравенства $(3.8)$ непрерывна на $\left(L^{p^{\prime}}(\Omega)\right)^{k}$. Поэтому само неравенство (3.8) справедливо для любого $v \in L^{p^{\prime}}(\Omega)^{k}$; следовательно,

$$
\varliminf_{\varepsilon \rightarrow 0} J_{\varepsilon} \geqslant \sup _{v \in\left(L^{p^{\prime}}(\Omega)\right)^{k}}\left[\int_{\Omega} \nabla u^{0} \cdot v d x-\int_{\Omega} f_{0}^{*}(v) d x\right]=\int_{\Omega} f_{0}\left(\nabla u^{0}\right) d x
$$

по классической теореме о сопряженном функционале [8, гл. IX]. Лемма доказана.

Теперь сравним энергии $m_{\varepsilon}$ и $m_{0}$ исходной и усредненной задач.

Лемма 3.4. Имеет место неравенство $\overline{\lim }_{\varepsilon \rightarrow 0} m_{\varepsilon} \leqslant m_{0}$.

ДокАЗАТЕльСТво. Для данного $\delta>0$ найдем функцию $u \in C_{0}^{\infty}(\Omega)$, такую, что

$$
\int_{\Omega}\left[f_{0}(\nabla u)+|u|^{p}-g u\right] d x \leqslant m_{0}+\delta .
$$

В силу оценки (1.2) и выпуклости имеем оценку

$$
|f(y, \xi)-f(y, \eta)| \leqslant c|\xi-\eta|\left(|\xi|^{p-1}+|\eta|^{p-1}\right)
$$

и аналогичную оценку для $f_{0}$. Фиксируем кусочно постоянный вектор $h(x)$, такой, что

$$
|f(y, \nabla u)-f(y, h)| \leqslant \tau, \quad\left|f_{0}(\nabla u)-f_{0}(h)\right| \leqslant \tau, \quad \tau=\delta|\Omega|^{-1} .
$$

Пусть $A_{j}$ - подобласти, где $h$ постоянен, $\left.h\right|_{A_{j}}=\xi^{j}, A_{j}$ достаточно гладкие. Рассмотрим вариационную задачу $(1.5)$ и выберем $v^{j}(y) \in C_{\mathrm{per}}^{\infty}$ так, что

$$
f_{0}\left(\xi^{j}\right)+\tau \leqslant \int_{\square} f\left(y, \xi^{j}+\nabla_{y} v^{j}(y)\right) d \mu .
$$

Положим

$$
u^{\varepsilon}(x)=u(x)+\varepsilon \sum \varphi_{j}(x) v^{j}\left(\varepsilon^{-1} x\right), \quad \varphi^{j} \in C_{0}^{\infty}\left(A_{j}\right), 0 \leqslant \varphi_{j} \leqslant 1,
$$

и рассмотрим интегралы

$$
\begin{aligned}
J_{j}^{\varepsilon} & =\int_{\bar{A}_{j}} f\left(\varepsilon^{-1} x, \nabla u^{\varepsilon}\right) d \mu_{\varepsilon} \\
& =\int_{\bar{A}_{j}} f\left(\varepsilon^{-1} x, \nabla u+\varphi_{j}(x) \nabla_{y} v^{j}\left(\varepsilon^{-1} x\right)+\varepsilon \nabla \varphi_{j}(x) v^{j}\left(\varepsilon^{-1} x\right)\right) d \mu_{\varepsilon} .
\end{aligned}
$$


В силу (3.10), (3.11), (1.11)

$$
\varlimsup_{\varepsilon \rightarrow 0} J_{j}^{\varepsilon} \leqslant \varlimsup_{\varepsilon \rightarrow 0} \int_{\bar{A}_{j}} f\left(\varepsilon^{-1} x, \xi^{j}+\varphi_{j}(x) \nabla_{y} v^{j}\left(\varepsilon^{-1} x\right)\right) d \mu_{\varepsilon}+\left|A_{j}\right| \tau .
$$

Далее по выпуклости и свойству среднего значения

$$
\begin{aligned}
f\left(\varepsilon^{-1} x\right. & \left., \xi^{j}+\varphi_{j}(x) \nabla_{y} v^{j}\left(\varepsilon^{-1} x\right)\right) \\
& \leqslant \varphi_{j} f\left(\varepsilon^{-1} x, \xi^{j}+\nabla_{y} v^{j}\left(\varepsilon^{-1} x\right)\right)+\left(1-\varphi_{j}\right) f\left(\varepsilon^{-1} x, \xi^{j}\right) \\
\varlimsup & J_{j}^{\varepsilon} \leqslant \int_{A_{j}} \varphi_{j} f_{0}\left(\xi^{j}\right) d x+\left(\int_{A_{j}}\left(1-\varphi_{j}\right) d x\right)\left(\int_{\square} f\left(y, \xi^{j}\right) d \mu\right)+\left|A_{j}\right| \tau \\
& \leqslant\left(f_{0}\left(\xi^{j}\right)+3 \tau\right)\left|A_{j}\right|
\end{aligned}
$$

при подходящем выборе функций $\varphi_{j}$. В результате получаем

$$
\begin{aligned}
\varlimsup_{\varepsilon \rightarrow 0} \int_{\Omega} f\left(\varepsilon^{-1} x, \nabla u^{\varepsilon}\right) d \mu_{\varepsilon} & \leqslant \sum \varlimsup \\
& =\int_{\Omega} f_{0}(h) d x+3 \delta \leqslant \int_{\Omega}^{\varepsilon} f_{0}(\nabla u) d x+4 \delta .
\end{aligned}
$$

Кроме того, из (3.12) ясно, что

$$
\lim _{\varepsilon \rightarrow 0} \int_{\Omega}\left|u^{\varepsilon}\right|^{p} d \mu=\int_{\Omega}|u|^{p} d x, \quad \lim _{\varepsilon \rightarrow 0} \int_{\Omega} g u^{\varepsilon} d \mu_{\varepsilon}=\int_{\Omega} g u d x .
$$

Поэтому (см. (3.9))

$$
\varlimsup_{\varepsilon \rightarrow 0} m_{\varepsilon} \leqslant \varlimsup_{\varepsilon \rightarrow 0} \int_{\Omega}\left[f\left(\varepsilon^{-1} x, \nabla u^{\varepsilon}\right)+\left|u^{\varepsilon}\right|^{p}-g u^{\varepsilon}\right] d \mu_{\varepsilon} \leqslant m_{0}+5 \delta,
$$

и лемма доказана.

3. Теперь мы в состоянии доказать основную теорему 1.2 .

Рассмотрим решение $u^{\varepsilon}$ исходной задачи (1.3). Оценки из (3.1) выполняются (см. (2.1)); можно также считать, что $u^{\varepsilon} \in C_{0}^{\infty}$, заменив при необходимости $u^{\varepsilon}$ гладкими приближениями (см. (2.2)). Пусть $u^{0}-$ предельная точка последовательности $u^{\varepsilon}$ в смысле (3.2). Тогда по леммам 3.1 и 3.3

$$
\begin{aligned}
\lim _{\varepsilon \rightarrow 0} m_{\varepsilon} & \geqslant \frac{\lim }{\varepsilon \rightarrow 0} \int_{\Omega} f\left(\varepsilon^{-1} x, \nabla u^{\varepsilon}\right) d \mu_{\varepsilon}+\underline{\lim }_{\varepsilon \rightarrow 0} \int_{\Omega}\left|u^{\varepsilon}\right|^{p} d \mu_{\varepsilon}-\int_{\Omega} g u^{0} d x \\
& \geqslant \int_{\Omega}\left[f_{0}\left(\nabla u^{0}\right)+\left|u^{0}\right|^{p}-g u^{0}\right] d x \geqslant m_{0} .
\end{aligned}
$$

На последнем шаге использовался тот факт, что $u^{0} \in S_{0}$. Из леммы 3.4 получаем, что в (3.13) должны наблюдаться строгие равенства, т.е. $u^{0}$ решение усредненной задачи. Отсюда же получаются и все утверждения теоремы 1.2 . 


\section{§4. Некоторые замечания}

1. Доказательство леммы 3.3 опиралось на двойственное представление для усредненного лагранжиана. Покажем, что в случае $f=|\xi|^{p}$ можно обойтись без двойственности. Исходя из (1.7), имеем

$$
\Phi(\xi)=\min _{v \in X}\left\langle|\xi+v|^{p}\right\rangle,
$$

$X$ - замыкание множества $\left\{\nabla \varphi, \varphi \in C_{\mathrm{per}}^{\infty}\right\}$ в $L^{p}(\square, d \mu)^{N}$.

Задача (4.1) обладает единственным решением $v \in X$, и для этого решения выполнено уравнение Эйлера:

$$
\left\langle|\xi+v|^{p-2}(\xi+v) \cdot \nabla \varphi\right\rangle=0 \quad \forall \varphi \in C_{\text {per }}^{\infty} .
$$

Если $v, \tilde{v}$ - решения этого уравнения для $\xi, \tilde{\xi} \in \mathbb{R}^{N}$, то по выпуклости

$$
\begin{aligned}
\Phi(\tilde{\xi})-\Phi(\xi) & =\left\langle|\tilde{\xi}+\tilde{v}|^{p}-|\xi+v|^{p}\right\rangle \geqslant p\left\langle|\xi+v|^{p-2}(\xi+v) \cdot(\tilde{\xi}-\xi+\tilde{v}-v)\right\rangle \\
& =p\left\langle|\xi+v|^{p-2}(\xi+v)\right\rangle \cdot(\tilde{\xi}-\xi)
\end{aligned}
$$

в силу (4.2). Отсюда следует, что

$$
\Phi^{\prime}(\xi)=p\langle z\rangle, \quad \text { где } z=|\xi+v|^{p-2}(\xi+v) .
$$

Ясно, что $\Phi^{\prime}(\xi) \in E$ (это следствие равенства $\Phi(\xi+q)=\Phi(\xi)$ для $q \in E^{\perp}$ ). Кроме того, поскольку $\Phi$ коэрцитивен на $E$, то $\Phi^{\prime}(\xi)$ пробегает все пространство $E$, когда $\xi$ пробегает $\mathbb{R}^{N}$. Отметим, что вектор $z$ из (4.3) соленоидален в силу $(4.2)$ и что $\Phi(\xi)=\left\langle|z|^{p^{\prime}}\right\rangle$.

Нам нужно доказать неравенство $(3.7)$, в котором $f_{0}=\Phi$, для функции $u^{\varepsilon}$, удовлетворяющей условиям (3.1), (3.2). Возьмем $\xi \in \mathbb{R}^{N}$ и соответствующий вектор $z$ из (4.3). Для $z_{\varepsilon}(x)=z\left(\varepsilon^{-1} x\right)$ по неравенству Юнга

$$
\int_{\bar{A}}\left|\nabla u^{\varepsilon}\right|^{p} d \mu_{\varepsilon} \geqslant p \int_{\bar{A}} \varphi \nabla u^{\varepsilon} \cdot z_{\varepsilon} d \mu_{\varepsilon}-(p-1) \int_{\bar{A}} \varphi\left|z_{\varepsilon}\right|^{p^{\prime}} d \mu_{\varepsilon}
$$

где $A$ - липшицева подобласть в $\Omega, \varphi \in C_{0}^{\infty}(A), 0 \leqslant \varphi \leqslant 1$.

По лемме 3.2 и свойству среднего значения

$$
\varliminf_{\varepsilon \rightarrow 0} \int_{\bar{A}}\left|\nabla u^{\varepsilon}\right|^{p} d \mu_{\varepsilon} \geqslant \int_{A} \varphi \nabla u^{0} \cdot \Phi^{\prime}(\xi) d x-(p-1) \int_{A} \varphi \Phi(\xi) d x .
$$

Taк как $\nabla u^{0} \cdot \Phi^{\prime}(\xi) \in L^{p}(\Omega)$, то

$$
\varliminf_{\varepsilon \rightarrow 0} \int_{\bar{A}}\left|\nabla u^{\varepsilon}\right|^{p} d \mu_{\varepsilon} \geqslant \int_{A} \nabla u^{0} \cdot \Phi^{\prime}(\xi) d x-(p-1) \int_{A} \Phi(\xi) d x .
$$

Отсюда заключаем, что

$$
\varliminf_{\varepsilon \rightarrow 0} \int_{\Omega}\left|\nabla u^{\varepsilon}\right|^{p} d \mu_{\varepsilon} \geqslant \int_{\Omega} \nabla u^{0} \cdot \Phi^{\prime}(v) d x-(p-1) \int_{\Omega} \Phi(v) d x
$$

для любого финитного кусочно постоянного вектора $v(x)$. 
Остается убедиться (предельным переходом), что в (4.4) можно положить $v=\nabla u^{0}$, что сразу даст искомое неравенство $(3.7)$, так как $\nabla u^{0} \cdot \Phi^{\prime}\left(\nabla u^{0}\right)=$ $p \Phi\left(\nabla u^{0}\right)$.

«Укороченный» градиент $\chi=\left\{\partial u^{0} / \partial x_{1}, \ldots, \partial u^{0} / \partial x_{k}\right\}$ принадлежит $\left(L^{p}(\Omega)\right)^{k}$ и $\left|\Phi^{\prime}(\xi)\right| \leqslant c|\xi|^{p-1}$. Поэтому, беря кусочно-постоянные $v_{n}$ со значениями в $\mathbb{R}^{k}, v_{n} \rightarrow \chi$ в $\left(L^{p}(\Omega)\right)^{k}$, получим, что $\Phi^{\prime}\left(v_{n}\right) \rightarrow \Phi^{\prime}\left(\nabla u^{0}\right)$ в $\left(L^{p^{\prime}}(\Omega)\right)^{k}$ и предельный переход в (4.4) возможен.

2. О коэрцитивности усредненного лагранжиана. Введем следующее

ОПрЕДЕЛЕниЕ 4.1. Периодическая мера $\mu$ называется $p$-связной в $\mathbb{R}^{N}$, если $u(x) \equiv$ const $\mu$-п.в., как только найдутся $u_{n} \in C^{\infty}\left(\mathbb{R}^{N}\right)$, такие, что

$$
u_{n} \rightarrow u, \quad \nabla u_{n} \rightarrow 0 \text { в } L_{\mathrm{loc}}^{p}\left(\mathbb{R}^{N}, d \mu\right) .
$$

Очевидно, что $p$-связность в $\mathbb{R}^{N}$ влечет за собой $p$-связность (на торе). Имеется естественная гипотеза, что $p$-связность в $\mathbb{R}^{N}$ обеспечивает коэриитивность лагранжиана $\Phi$, но она остается недоказанной в полном объеме. Имеет место следующий частный результат:

ТЕорема 4.2. Пусть мера $\mu$ является $p$-связной в $\mathbb{R}^{N}$. Если дополнительно она симметрична относительно координатных осей, то усредненньий лагранжиан коэриитивен.

Эта теорема доказана в [1] для случая перфорированных областей, т.е. для меры (1.10). Доказательство остается в силе и для общей меры.

В частности, усредненный лагранжиан коэрцитивен, если мера $\mu$ является $p$-связной в $\mathbb{R}^{N}$ и

$$
\mu \leqslant C \mu_{i}, \quad i=1, \ldots, N,
$$

где $\mu_{i}$ - симметризация $\mu$ относительно оси $x_{i}$.

3. Для приложений к эволюционным уравнениям важно изучить несколько иную по сравнению с (1.3) задачу, а именно,

$$
m_{\varepsilon}=\inf _{u \in C_{0}^{\infty}(\Omega)} \int_{\Omega}\left(f\left(\varepsilon^{-1} x, \nabla u\right)+|u|^{q}-g u\right) d \mu_{\varepsilon},
$$

где показатель $q>1$ никак не связан с показателем $p$ из (1.2). При этом наиболее важен случай $q=2$. Решение задачи (4.5) сушествует и единственно в пространстве

$$
V_{\varepsilon}=\text { замыкание }\left\{(u, \nabla u), \text { где } u \in C_{0}^{\infty}(\Omega)\right\} \text { в } L^{q}\left(\Omega, d \mu_{\varepsilon}\right) \times L^{p}\left(\Omega, d \mu_{\varepsilon}\right)^{N} .
$$

Усредненная задача имеет вид (1.4), где слагаемое $|u|^{p}$ заменено на $|u|^{q}$, a усредненный лагранжиан $f_{0}$ прежний. Формулировка теоремы усреднения сохраняется с единственным изменением:

$$
\int_{\Omega}\left|u^{\varepsilon}\right|^{q} d \mu_{\varepsilon} \rightarrow \int_{\Omega}\left|u^{0}\right|^{q} d x .
$$

Доказательство в целом остается прежним. Некоторую проблему составляет только лемма 3.2, где мы существенно использовали тот факт, что в 
условии (3.1) показатели для $u^{\varepsilon}$ и $\nabla u^{\varepsilon}$ одинаковы. Сейчас мы докажем соответствующее обобщение леммы 3.2 .

Пусть

$$
u^{\varepsilon} \in C_{0}^{\infty}(\Omega), \quad \int_{\Omega}\left|u^{\varepsilon}\right|^{q} d \mu_{\varepsilon} \leqslant \alpha<\infty, \quad \int_{\Omega}\left|u^{\varepsilon}\right|^{p} d \mu_{\varepsilon} \leqslant \beta<\infty .
$$

Без ограничения общности (см. лемму 3.1) считаем, что

$$
\lim _{\varepsilon \rightarrow 0} \int_{\Omega} \varphi u^{\varepsilon} d \mu_{\varepsilon}=\int_{\Omega} \varphi u^{0} d x \quad\left(\forall \varphi \in C^{\infty}(\bar{\Omega})\right), u^{0} \in L^{q}(\Omega) .
$$

Рассмотрим гладкие нечетные функции $h_{n}(t)$ на числовой оси, такие, что

$$
\begin{array}{lll}
h_{n}(t)=t & \text { при } 0 \leqslant t \leqslant n, & 0 \leqslant h_{n}^{\prime}(t) \leqslant 1, \\
h_{n}(t)=2 n & \text { при } t \geqslant 2 n, & h(t) \leqslant t \text { при } t \geqslant 0,
\end{array}
$$

и положим $u^{\varepsilon, n}=h_{n}\left(u^{\varepsilon}\right)$. Пусть

$$
K_{\varepsilon, n}=\left\{x \in \Omega,\left|u^{\varepsilon}\right| \geqslant n\right\} ;
$$

заметим, что

$$
\mu_{\varepsilon}\left(K_{\varepsilon, n}\right) \leqslant \frac{1}{n^{q}} \int_{\Omega}\left|u^{\varepsilon}\right|^{q} d \mu_{\varepsilon} \leqslant \frac{\alpha}{n^{q}} .
$$

Обозначим через $u^{0, n}$ слабый предел (в смысле (4.7)) $u^{\varepsilon, n}$ при фиксированном $n$. Поскольку $\left|u^{\varepsilon, n}\right| \leqslant 2 n,\left|\nabla u^{\varepsilon, n}\right| \leqslant\left|\nabla u^{\varepsilon}\right|$, по лемме 3.2

$$
\begin{gathered}
\lim _{\varepsilon \rightarrow 0} \int_{\Omega} \varphi \nabla u^{\varepsilon, n} \cdot z_{\varepsilon} d \mu_{\varepsilon}=\int_{\Omega} \varphi \nabla u^{\varepsilon, n} \cdot \eta d x, \\
\int_{\Omega}\left|\nabla u^{0, n} \cdot \eta\right|^{p} d x \leqslant \beta\left\langle|z|^{p^{\prime}}\right\rangle^{p-1} .
\end{gathered}
$$

Считаем, что $\max |\varphi|=1$, и учитываем, что $\left|u^{\varepsilon}-u^{\varepsilon, n}\right| \leqslant\left|u^{\varepsilon}\right|$. Тогда

$$
\begin{aligned}
\left|\int_{\Omega} \varphi\left(u^{\varepsilon}-u^{\varepsilon, n}\right) d \mu_{\varepsilon}\right| & \leqslant \alpha^{1 / q} \mu_{\varepsilon}^{1 / q^{\prime}}\left(K_{\varepsilon, n}\right) \leqslant \frac{\alpha^{(q+1) / q}}{n^{q}}, \\
\left|\int_{\Omega} \varphi\left(u^{0}-u^{0, n}\right) d x\right| & \leqslant \frac{\alpha^{(q+1) / q}}{n^{q}} .
\end{aligned}
$$

Отсюда следует, что $u^{0, n} \rightarrow u^{0}$ слабо в $L^{q}(\Omega)$. Тогда оценка (4.9) дает слабую сходимость $\nabla u^{\varepsilon, n} \cdot \eta \rightarrow \nabla u^{0} \cdot \eta$ в $L^{p}(\Omega)$ и поэтому

$$
\lim _{n \rightarrow \infty} \int_{\Omega} \varphi u^{0, n} \cdot \eta d x=\int_{\Omega} \varphi \nabla u^{0} \cdot \eta d x
$$

Остается для разности

$$
J_{\varepsilon, n}=\int_{\Omega} \varphi\left(\nabla u^{\varepsilon}-\nabla u^{\varepsilon, n}\right) z_{\varepsilon} d \mu_{\varepsilon}, \quad|\varphi| \leqslant 1
$$


доказать оценку вида

$$
\left|J_{\varepsilon, n}\right| \leqslant c_{n}, \quad \text { где } c_{n} \rightarrow 0 \text { при } n \rightarrow \infty .
$$

Тогда искомое равенство (3.4) будет очевидным в силу (4.8), (4.10). Так как $\left|\nabla u^{\varepsilon}-\nabla u^{\varepsilon, n}\right| \leqslant\left|\nabla u^{\varepsilon}\right|$, то

$$
\left|J_{\varepsilon, n}\right| \leqslant \beta^{1 / p}\left(\int_{K_{\varepsilon, n}}\left|z_{\varepsilon}\right|^{p^{\prime}} d \mu_{\varepsilon}\right)^{1 / p^{\prime}}
$$

Имеем $a(x)=|z(x)|^{p^{\prime}}, a \in L^{1}(\square, d \mu)$. Найдем коэрцитивную функцию $\gamma(t)$ на оси (т.е. $\gamma(t)$ четна, непрерывна и $\left.\lim _{t \rightarrow+\infty} t^{-1} \gamma(t)=+\infty\right)$, такую, что $\gamma(a) \in L^{1}(\square, d \mu)$. Сопряженная функция $\gamma^{*}(t)=\sup _{s}(t s-\gamma(s))$ автоматически коэрцитивна. Функцию $\gamma$ считаем выпуклой, заменив при необходимости ее на вторую сопряженную $\gamma^{* *}$. По неравенству Юнга при $s \geqslant 1$

$$
\begin{gathered}
a_{\varepsilon} \gamma\left(s^{-1} a_{\varepsilon}\right)+\gamma^{*}(s) \leqslant s^{-1} \gamma\left(a_{\varepsilon}\right)+|\gamma(0)|+\gamma^{*}(s) \\
I_{\varepsilon, n}=\int_{K_{\varepsilon, n}} a_{\varepsilon} d \mu_{\varepsilon} \leqslant s^{-1} \int_{\Omega} \gamma\left(a_{\varepsilon}\right) d \mu_{\varepsilon}+(|\gamma(0)|+\gamma(s)) \mu_{\varepsilon}\left(K_{\varepsilon, n}\right) \\
\leqslant 2 s^{-1}|\Omega|\langle\gamma(a)\rangle+\frac{\left(|\gamma(0)|+\gamma^{*}(s)\right) \alpha}{n^{q}}
\end{gathered}
$$

при достаточно малом $\varepsilon$. Поскольку $s$ произвольно, то $I_{\varepsilon, n} \leqslant l_{n}\left(\right.$ при $\left.\varepsilon \leqslant \varepsilon_{0}\right)$ и $l_{n} \rightarrow 0$ при $n \rightarrow \infty$. Отсюда и из (4.12) следует искомая оценка (4.11).

\section{ЛитЕРАТУРА}

1. Жиков В. В. Связность и усреднение. Примеры фрактальной проводимости. Матем. сб., 187, № 8, 3-40 (1996).

2. De Arcangelis R., Serra Cassano F. On the homogenization of degenerate elliptic equation in divergence form. J. Math. Pures Appl., 71, 1-20 (1992).

3. Жиков В. В., Козлов С. М., Олейник О. А. Усреднение дифференциальных операторов. Наука, М., 1993.

4. Jikov V. V., Kozlov S. M., Oleinik O. A. Homogenization of differential operators and integral functionals. Springer-Verlag, 1994.

5. Олейник О. А., Йосифьян Г. А., Шамаев А. С. Математические задачи теории сильно неоднородных сред. МГУ, М., 1990.

6. Жиков В. В. Усреднение в перфорированных случайных областях общего вида. Матем. заметки, 53, № 1, 41-58 (1993).

7. Zhikov $V$. $V$. On the homogenization of nonlinear variational problems in perforated domains. Russian J. Math. Phys., 2, No. 3, 393-408 (1994).

8. Экланд H., Темам P. Выпуклый анализ и вариационные проблемы. Мир, М., 1979.

Владимирский государственный

Поступило в редакцию педагогический университет

26 марта 1998 г.

e-mail: zhikov@vgpu.elcom.ru 